\title{
CRESCIMENTO DE FEIJÃO-DE-PORCO NA PRESENÇA DE CHUMBO ( ${ }^{\mathbf{1}}$ )
}

\author{
ELLEN LOREGIAN DE ALMEIDA $\left({ }^{2}\right)$; FERNANDA CASTRO CORREIA MARCOS $\left({ }^{2}\right)$; \\ MARLENE APARECIDA SCHIAVINATO $\left({ }^{3}\right)$; ANA MARIA MAGALHÃES ANDRADE LAGÔA $\left({ }^{4}\right)$; \\ MÔNICA FERREIRA DE ABREU ( $\left(^{5}\right)$
}

\begin{abstract}
RESUMO
O excesso de metais pesados no solo pode se tornar um perigo para a saúde do homem, dos animais e das plantas. A fitorremediação é uma técnica com aplicação crescente em áreas de solos poluídos visando à descontaminação do solo e da água, utilizando plantas como agente descontaminador. O presente trabalho teve por objetivo avaliar o potencial fitoextrator da espécie leguminosa feijão-de-porco [Canavalia ensiformis (L.) D.C.] em relação ao chumbo (Pb), por meio da avaliação dos efeitos desse metal pesado na fisiologia da planta e na nodulação das raízes. O experimento foi realizado sob condições naturais de luz e temperatura, em casa de vegetação, com vasos contendo $2 \mathrm{~L}$ de areia. As plantas, com e sem inoculação de rizóbio, foram submetidas a quatro concentrações de $\mathrm{Pb}\left(0,250,500\right.$ e $\left.1000 \mu \mathrm{mol} \mathrm{L}^{-1}\right)$. Verificou-se que o desenvolvimento das plantas de feijão-de-porco não foi inibido e não houve nas plantas sintomas de fitotoxicidade na parte aérea. Porém, as plantas não tiveram produção de nódulos radiculares. Observou-se, ainda, que nessa espécie ocorreram baixos índices de translocação de $\mathrm{Pb}$ para a parte aérea. Concluiu-se que as plantas de feijão-de-porco têm potencial para a fitoextração de $\mathrm{Pb}$, atingindo $400 \mathrm{mg}$ $\mathrm{Pb} \mathrm{kg}^{-1}$ nas raízes, e que a fixação de $\mathrm{N}$ por meio de nódulos foi afetada pela presença de $\mathrm{Pb}$.
\end{abstract}

Palavras-chave: fitorremediação, Canavalia ensiformis, feijão-de-porco, nódulos radiculares, rizóbio.

\section{ABSTRACT \\ GROWTH OF JACKBEAN IN THE PRESENCE OF LEAD}

Excessive soil metal concentrations may be hazardous for human, animal and plant health. Phytoremediation is a technique based on the use of plants as decontaminator agent and has been increasingly applied with the objective of soil and water remediation in polluted areas. The objective of the present work was to study a leguminous species - jackbean [Canavalia ensiformis (L.)D.C.] - as Pbphytoextractor through the evaluation of $\mathrm{Pb}$ effects on the plant physiology and root nodulation. The experiment was carried out under natural variation of light and temperature, in pots containing $2 \mathrm{~L}$ of sand under greenhouse conditions. Plants were treated with four Pb concentrations (0, 250, 500 and 1000 $\left.\mu \mathrm{mol} \mathrm{L}{ }^{-1}\right)$ in the presence and absence of rhizobium stirp. Jackbean plant development was not affected by $\mathrm{Pb}$ treatments and $\mathrm{Pb}$-toxicity symptoms were not observed. However, plants were not able to produce root nodules. Jackbean plants exibited low shoot/root $\mathrm{Pb}$ translocation indexes. Despite the fact that root nodulation and $\mathrm{N}$ fixation can be drastically reduced in the presence of high $\mathrm{Pb}$ concentrations, it was concluded that jackbean plants can be considered potential $\mathrm{Pb}$ phytoextrators, since root $\mathrm{Pb}$ concentrations reached up to $400 \mathrm{mg} \mathrm{kg}^{-1}$.

Key words: phytoremediation, Canavalia ensiformis, jackbeans, root nodules, rhizobium.

$\left({ }^{1}\right)$ Recebido para publicação em 3 de abril de 2007 e aceito em 31 de março de 2008. Extraído da Dissertação de Mestrado apresentada pela primeira autora ao Programa de Pós-Graduação em Agricultura Tropical e Subtropical, Instituto Agronômico (IAC), Campinas (SP).

$\left(^{2}\right)$ Graduanda do Curso de Graduação em Ciências Biológicas da Universidade Estadual de Campinas. Estagiária do Departamento de Fisiologia Vegetal, Campinas (SP). Bolsista do CNPq.

$\left({ }^{3}\right)$ Departamento de Fisiologia Vegetal, Instituto de Biologia, Unicamp, Campinas (SP).

$\left({ }^{4}\right)$ Centro de Ecofisiologia e Biofísica, IAC, Caixa Postal 28, 13012-970 Campinas (SP). E-mail: alagoa@iac.sp.gov.br $\left({ }^{*}\right)$ Autora correspondente.

$\left({ }^{5}\right)$ Centro de Solos e Recursos Agroambientais, IAC, Campinas (SP). E-mail: monica@iac.sp.gov.br 


\section{INTRODUÇÃO}

O crescente aumento da população ao longo dos últimos anos ocorreu de forma desorganizada e com deficitária infra-estrutura, resultando em intensas mudanças no meio ambiente, principalmente na degradação de áreas florestais, na poluição do solo e de mananciais e na contaminação de extensas áreas por resíduos que podem conter grandes quantidades de metais pesados (MPs) (IBGE, 2006). A contaminação do solo por metais tóxicos freqüentemente é resultado de atividades humanas, especialmente aquelas relacionadas com mineração; emissão, disposição ou vazamento de resíduos industriais; aplicação de lodo de esgoto; fertilizantes e pesticidas no meio agrícola (Malavolta, 1994). A preocupação ambiental em relação às áreas contaminadas por MPs é agravada quando esses entram na cadeia alimentar (ABReu et al., 1995). Segundo ATSDR (2006), existe nos Estados Unidos, uma lista contendo 275 substâncias orgânicas e inorgânicas consideradas perigosas, baseando-se na combinação de sua freqüência, toxicidade e potencial de exposição humana. $\mathrm{O}$ chumbo $(\mathrm{Pb})$ está presente nesta lista em segundo lugar.

A remediação de áreas contaminadas é uma exigência legal e um compromisso social que precisam ser executados, necessitando de grande demanda de tecnologia. As tecnologias efetivas e econômicas para remediação das áreas contaminadas por MPs ainda permanecem indefinidas. A que se destaca é a fitorremediação, que promove a desintoxicação do local ou remove os elementos contaminantes do solo (AcCioly e Siqueira, 2000). A fitorremediação tem como objetivo descontaminar o solo e a água utilizando-se plantas como agente descontaminador (NEWMAN et al., 1998). Segundo Pires et al. (2005), as técnicas para despoluir áreas contaminadas por diversos compostos orgânicos devem possuir: eficiência na descontaminação, simplicidade na execução, baixo tempo demandado pelo processo e baixo custo. $\mathrm{O}$ estudo e a utilização da fitoextração, um tipo de fitorremediação, precisam ser intensificados no Brasil, sendo necessárias mais pesquisas que utilizem espécies de clima tropical como remediadoras de áreas contaminadas.

A absorção de nutrientes pela maioria das plantas com sementes é bastante favorecida pela ocorrência de bactérias e fungos associados ao sistema radicular (LASAT, 2002). As bactérias do gênero Rhizobium são fixadoras de nitrogênio e vivem em simbiose com as espécies leguminosas, formando nódulos em suas raízes, capazes de absorverem o nitrogênio da atmosfera, sintetizando substâncias nitrogenadas, também utilizadas pela planta hospedeira. Em contrapartida, a leguminosa fornece açúcares e outros compostos orgânicos às bactérias presentes em seus nódulos (KerbauY, 2004).

A hipótese deste trabalho é que a espécie Canavalia ensiformis tem potencial fitoextrator em relação ao $\mathrm{Pb}$. O objetivo geral foi testar a hipótese avaliando os efeitos desse metal pesado na fisiologia da planta, considerando a tolerância da leguminosa ao $\mathrm{Pb}$, a influência de $\mathrm{Pb}$ na formação de nódulos radiculares e na fixação de $\mathrm{N}_{2}$ e o acúmulo de $\mathrm{Pb}$ nas raízes e na parte aérea.

\section{MATERIAL E MÉTODOS}

O experimento foi desenvolvido em casa de vegetação localizada nas dependências do Departamento de Fisiologia Vegetal da Universidade Estadual de Campinas (UNICAMP), sob condições de luz e temperatura naturais, no período de 23 de agosto a 9 de outubro de 2006 .

A espécie vegetal estudada foi a leguminosa herbácea Canavalia ensiformis (L.) D.C. (feijão-deporco), cujas sementes foram obtidas no Instituto Agronômico (IAC). O delineamento estatístico foi inteiramente casualizado, em esquema fatorial de 2 $x 4$, sendo dois tratamentos, com (C/R) e sem (S/R) inoculação de estirpes de rizóbio, e quatro concentrações de $\mathrm{Pb}\left(0,250,500\right.$ e $\left.1000 \mu \mathrm{mol} \mathrm{L}^{-1}\right)$. Para cada concentração foram consideradas sete repetições (plantas). Foi utilizada a solução nutritiva de HoAgLAND e ARNON (1939) modificada, contendo ou não $\mathrm{N}$ mineral. A solução era composta pelos seguintes nutrientes, com as respectivas concentrações $\left(\mathrm{mg} \mathrm{L}^{-1}\right): \mathrm{N}-\mathrm{NO}_{3}=196 ; \mathrm{P}=1,20 ; \mathrm{K}=$ $461 ; \mathrm{Ca}=360 ; \mathrm{Mg}=48 ; \mathrm{S}^{-\mathrm{SO}_{4}}=64 ; \mathrm{B}=0,46 ; \mathrm{Cu}=$ 0,$02 ; \mathrm{Fe}=2,50 ; \mathrm{Mn}=0,50 ; \mathrm{Zn}=0,05$.

As sementes foram previamente desinfetadas e colocadas para germinar em bandejas plásticas contendo areia lavada e esterilizada. Após a emissão da folha primária, as plântulas foram retiradas das bandejas e transferidas para vasos com capacidade de $2 \mathrm{~L}$ (uma planta/vaso), contendo areia como substrato, onde permaneceram até o momento da coleta. Durante o transplantio, metade das plantas foi submetida à inoculação com suspensão de uma estirpe específica de bactérias do gênero Rhizobium (VINCENT, 1970), previamente selecionada (ZANELLA, 2004). Após cinco dias, as plantas receberam doses únicas de $200 \mathrm{~mL}$ de solução contendo as diferentes concentrações de $\mathrm{Pb}$, utilizando-se como fonte de contaminação o acetato de chumbo $\left[\mathrm{Pb}\left(\mathrm{CH}_{3} \mathrm{COO}\right)_{2} \cdot 3 \mathrm{H}_{2} \mathrm{O}\right]$.

As plantas foram regadas uma vez por semana com $100 \mathrm{~mL}$ de solução nutritiva. Nos tratamentos com rizóbio, as plantas receberam solução nutritiva sem 
acréscimo de $\mathrm{N}$ mineral. A coleta das plantas foi realizada em três datas: 14,22 e 27 dias após a aplicação de $\mathrm{Pb}\left(1 .{ }^{\mathrm{a}},{ }^{2} .^{\mathrm{a}}\right.$ e $3 .^{\mathrm{a}}$ coletas respectivamente). Em cada coleta, foram realizadas as seguintes avaliações: altura da planta; número de folhas; massa seca das lâminas foliares, do caule mais pecíolos e das raízes. As medidas de área foliar foram feitas com um planímetro digital modelo LI -3100 (Licor).

Para a quantificação de $\mathrm{Pb}$, amostras de tecido vegetal foram digeridas por via úmida com aquecimento por microondas, utilizando $500 \mathrm{mg}$ (com precisão de $1 \mathrm{mg}$ ) do material seco e moído em tubos especiais de Teflon (X-Press). Em seguida, as amostras foram digeridas com uma mistura de $5 \mathrm{~mL}$ de ácido nítrico (70\%) e $3 \mathrm{~mL}$ de peróxido de hidrogênio (30\%) em forno de microondas especial (marca CEM-MarsXpress). O aparelho foi programado na potência 1200 $\mathrm{W}$, ficando 15 minutos a $200^{\circ} \mathrm{C}, 1$ minuto a $210^{\circ} \mathrm{C}$ e 1 minuto a $220^{\circ} \mathrm{C}$. Após a digestão, as amostras foram filtradas e colocadas em balão volumétrico de $25 \mathrm{~mL}$ e o volume final completado com água deionizada. A determinação do $\mathrm{Pb}$ foi feita por espectrometria de emissão óptica em plasma de argônio (ICP-OES-Varian Vista MPX).

Os teores de nitrogênio da parte aérea (folhas, caules e pecíolos) foram determinados pelo método de destilação Kjeldahl (ABreu et al., 2006).

$\mathrm{O}$ índice de translocação de chumbo (IT) foi calculado com base na relação entre a quantidade de metal acumulada na parte aérea e a acumulada na planta, de acordo com ABICHeQuer e BoHNEn (1998). Calculou-se, também, o índice de produção relativa (IP), pela relação (\%) entre a quantidade de matéria seca produzida na presença do metal e a produzida na ausência do metal.

Os dados foram submetidos à análise de variância (teste F) e, quando houve significância estatística ( $5 \%$ de probabilidade), foram realizadas análises de regressão polinomial (linear e quadrática).

\section{RESULTADOS E DISCUSSÃO}

Em plantas de feijão-de-porco foram observados sintomas de fitotoxidade só nas raízes. Apesar da inoculação realizada nas plantas $C / R$, não houve formação de nódulos radiculares em nenhum dos tratamentos. Observou-se, ao longo de todo o experimento, nas plantas do tratamento C/ $\mathrm{R}$, uma coloração verde mais clara quando comparadas com as do tratamento sem rizóbio (S/ $\mathrm{R})$, indicando possível falta de nitrogênio em relação a estas, que receberam solução nutritiva contendo $\mathrm{N}$ mineral.
Nas medidas de altura e do número de folhas das plantas expostas ao $\mathrm{Pb}$ por 14, 22 e 27 dias não houve diferenças significativas quando comparadas aos tratamentos $S$ / R e C/R bem como em função das doses de $\mathrm{Pb}$ aplicadas (tabela 1). Em plantas de Carex rostrata, Eriophorum angustifolium e Phragmites australis não houve modificação no crescimento na presença de Pb (Stoltz e Greger, 2002), resultados semelhantes aos constatados neste experimento.

Segundo Punz e Sieghardt (1993), em algumas plantas observaram-se murchamento e posterior queda de folhas quando submetidas a altas concentrações de $\mathrm{Pb}$. Contudo, neste experimento esse fato não ocorreu, o número de folhas continuou constante à medida que se aumentava a dose de $\mathrm{Pb}$. Não se observaram sintomas de fitotoxicidade como murchamento, amarelamento das folhas, clorose, necrose e queda.

Nas plantas S/R e C/R, não houve diferenças nas medidas de área foliar com o aumento das doses de $\mathrm{Pb}$ aplicadas; houve, porém, diferenças significativas entre esses tratamentos em todos dias analisados (Tabela 1).

A diminuição na área foliar tem sido considerada um dos efeitos mais significativos da deficiência do nitrogênio (Vos e Van Der Putten, 1998). Neste experimento, observou-se menor área foliar nas plantas $C / R$ o que pode ser justificado pelo fato dessas plantas não terem recebido $\mathrm{N}$ mineral e nem terem assimilado $\mathrm{N}$ proveniente da fixação de $\mathrm{N}_{2}$, visto que não houve formação de nódulos radiculares.

Plantas de mamão cultivadas sob deficiência de nitrogênio reduziram a área de interceptação da energia solar incidente, minimizando a redução da concentração de nitrogênio foliar. Com essa estratégia, essas plantas minimizam o efeito negativo sobre a capacidade fotossintética por unidade de área foliar, evidenciado pela pequena redução da fotossíntese (CRUz et al., 2007). Estratégia semelhante é utilizada por plantas de batata (Vos e Van Der Putten, 1998). Em milho, a prioridade é minimizar reduções na área foliar e na interceptação de luz, em detrimento da concentração de nitrogênio foliar, ocasionando maior redução da atividade fotossintética por unidade de área (Vos et al., 2005).

Na matéria seca de raiz em plantas aos 14, 22 e 27 dias após a aplicação de $\mathrm{Pb}$ não houve diferenças significativas entre os tratamentos C/R e S/R (Tabela 2). A matéria seca da raiz também não foi afetada pelas doses de $\mathrm{Pb}$ aplicadas. Apesar disto, as raízes tiveram atrofia e escurecimento. 
Tabela 1. Altura, área foliar e número de folhas de plantas de feijão-de-porco [Canavalia ensiformis (L.) D.C.] expostas a diferentes doses de $\mathrm{Pb}$ por 14, 22 e 27 dias

\begin{tabular}{|c|c|c|c|c|c|c|}
\hline \multirow{2}{*}{ Concentração de $\mathrm{Pb}$} & $\mathrm{C} / \mathrm{R}$ & $\mathrm{S} / \mathrm{R}$ & $\mathrm{C} / \mathrm{R}$ & $\mathrm{S} / \mathrm{R}$ & $\mathrm{C} / \mathrm{R}$ & $\mathrm{S} / \mathrm{R}$ \\
\hline & \multicolumn{2}{|c|}{14 dias } & \multicolumn{2}{|c|}{22 dias } & \multicolumn{2}{|c|}{27 dias } \\
\hline$\mu \mathrm{mol} \mathrm{L}^{-1}$ & \multicolumn{6}{|c|}{ Altura $(\mathrm{cm})$} \\
\hline 0 & $24,2 \mathrm{a}$ & $38,2 \mathrm{a}$ & 52,9 a & $63,3 \mathrm{a}$ & $92,2 \mathrm{a}$ & $64,1 \mathrm{a}$ \\
\hline 250 & 25,7 a & $29,4 \mathrm{a}$ & 49,3 a & 49,9 a & 87,5 a & $64,7 \mathrm{a}$ \\
\hline 500 & $34,3 \mathrm{a}$ & $37,9 \mathrm{a}$ & $42,5 \mathrm{a}$ & 51,6 a & 90,5 a & $94,0 \mathrm{a}$ \\
\hline \multirow[t]{2}{*}{1000} & $23,1 \mathrm{a}$ & $30,8 \mathrm{a}$ & 47,7 a & $54,9 \mathrm{a}$ & $68,8 \mathrm{~b}$ & $83.2 \mathrm{a}$ \\
\hline & \multicolumn{6}{|c|}{ Área foliar $\left(\mathrm{cm}^{2}\right)$} \\
\hline 0 & $390,2 \mathrm{~b}$ & $607,5 \mathrm{a}$ & $584,3 \mathrm{~b}$ & $764,1 \mathrm{a}$ & $630,4 \mathrm{~b}$ & $848,9 \mathrm{a}$ \\
\hline 250 & $377,2 \mathrm{~b}$ & $503,9 \mathrm{a}$ & $558,9 \mathrm{~b}$ & $718,3 \mathrm{a}$ & $719,3 \mathrm{~b}$ & $820,2 \mathrm{a}$ \\
\hline 500 & $454,1 \mathrm{~b}$ & $556,5 \mathrm{a}$ & $566,9 \mathrm{~b}$ & $772,5 \mathrm{a}$ & $659.0 \mathrm{~b}$ & $955,9 \mathrm{a}$ \\
\hline \multirow[t]{2}{*}{1000} & $416,0 \mathrm{~b}$ & $476,3 \mathrm{a}$ & $572,8 \mathrm{~b}$ & 719,9 a & $600,5 \mathrm{~b}$ & $930,7 \mathrm{a}$ \\
\hline & \multicolumn{6}{|c|}{ Folhas $\left(\mathrm{n} .^{\circ}\right)$} \\
\hline 0 & $2,9 \mathrm{a}$ & $3,0 \mathrm{a}$ & $3,5 \mathrm{a}$ & $4,0 \mathrm{a}$ & $3,7 \mathrm{a}$ & $4,0 \mathrm{a}$ \\
\hline 250 & $2,9 \mathrm{a}$ & $2,8 \mathrm{a}$ & $3,0 \mathrm{a}$ & $3,3 \mathrm{a}$ & $4,0 \mathrm{a}$ & $4,3 \mathrm{a}$ \\
\hline 500 & $3,0 \mathrm{a}$ & $3,0 \mathrm{a}$ & $3,3 \mathrm{a}$ & $3,6 \mathrm{a}$ & $3,8 \mathrm{a}$ & $4,5 \mathrm{a}$ \\
\hline 1000 & $3,0 \mathrm{a}$ & $3,0 \mathrm{a}$ & $3,5 \mathrm{a}$ & $3,7 \mathrm{a}$ & $4,0 \mathrm{a}$ & $4,6 \mathrm{a}$ \\
\hline
\end{tabular}

Letras diferentes indicam diferenças estatísticas pelo teste Tukey a $5 \%$.

Letras minúsculas comparam $\mathrm{C} / \mathrm{R}$ e $\mathrm{S} / \mathrm{R}$ dentro de cada dia e letras maíusculas comparam as diferentes concentrações de Pb, em cada tratamento. Cada dado representa o valor médio de sete repetições.

Tabela 2. Massas secas das raízes, do caule e das folhas e teor de nitrogênio da parte aérea (caule + folhas) de plantas de feijão-de-porco [Canavalia ensiformis (L.) D.C.] expostas a diferentes doses de Pb por 14, 22 e 27 dias

\begin{tabular}{|c|c|c|c|c|c|c|}
\hline \multirow{2}{*}{ Concentração de $\mathrm{Pb}$} & $\mathrm{C} / \mathrm{R}$ & $S / R$ & $\mathrm{C} / \mathrm{R}$ & $S / R$ & $\mathrm{C} / \mathrm{R}$ & $\mathrm{S} / \mathrm{R}$ \\
\hline & \multicolumn{2}{|c|}{14 dias } & \multicolumn{2}{|c|}{22 dias } & \multicolumn{2}{|c|}{27 dias } \\
\hline$\mu \mathrm{mol} \mathrm{L}{ }^{-1}$ & \multicolumn{6}{|c|}{ Massa seca da raiz (mg) } \\
\hline 0 & $0,66 \mathrm{Aa}$ & $0,59 \mathrm{Aa}$ & $0,74 \mathrm{Aa}$ & $0,81 \mathrm{Aa}$ & $0,65 \mathrm{Aa}$ & $0,83 \mathrm{Aa}$ \\
\hline 250 & 0,59 Аа & $0,50 \mathrm{Aa}$ & $0,80 \mathrm{Aa}$ & $0,75 \mathrm{Aa}$ & $0,70 \mathrm{Aa}$ & $0,83 \mathrm{Aa}$ \\
\hline 500 & $0,55 \mathrm{Aa}$ & $0,59 \mathrm{Aa}$ & 0,75 Aa & $0,74 \mathrm{Aa}$ & $0,96 \mathrm{Aa}$ & $0,83 \mathrm{Aa}$ \\
\hline \multirow[t]{2}{*}{1000} & $0,65 \mathrm{Aa}$ & $0,55 \mathrm{Aa}$ & $0,77 \mathrm{Aa}$ & $0,75 \mathrm{Aa}$ & $0,77 \mathrm{Ab}$ & $1,08 \mathrm{Aa}$ \\
\hline & \multicolumn{6}{|c|}{ Massa seca do caule (mg) } \\
\hline 0 & $0,79 \mathrm{Ab}$ & $1,11 \mathrm{Aa}$ & $1,35 \mathrm{Ab}$ & $1,68 \mathrm{Aa}$ & $1,76 \mathrm{Aa}$ & $2,13 \mathrm{Aa}$ \\
\hline 250 & $0,80 \mathrm{Aa}$ & $0,99 \mathrm{Aa}$ & $1,30 \mathrm{Ab}$ & $1,65 \mathrm{Aa}$ & $1,88 \mathrm{Aa}$ & $1,93 \mathrm{Aa}$ \\
\hline 500 & $0,89 \mathrm{Ab}$ & $1,14 \mathrm{Aa}$ & $1,40 \mathrm{Aa}$ & $1,65 \mathrm{Aa}$ & $1,84 \mathrm{Aa}$ & $2,29 \mathrm{Aa}$ \\
\hline \multirow[t]{2}{*}{1000} & 0,77 Aa & 0,95 Аа & 1,49 Аа & $1,61 \mathrm{Aa}$ & $1,63 \mathrm{Ab}$ & $2,31 \mathrm{Aa}$ \\
\hline & \multicolumn{6}{|c|}{ Massa seca da folha (mg) } \\
\hline 0 & $1,49 \mathrm{Ab}$ & $1,89 \mathrm{Aa}$ & $2,12 \mathrm{Ab}$ & $2,71 \mathrm{Aa}$ & $2,53 \mathrm{Ab}$ & $3,30 \mathrm{Aa}$ \\
\hline 250 & $1,49 \mathrm{Aa}$ & $1,65 \mathrm{Aa}$ & $2,10 \mathrm{Aa}$ & $2,39 \mathrm{Aa}$ & $2,80 \mathrm{Aa}$ & $3,10 \mathrm{Aa}$ \\
\hline 500 & $1,58 \mathrm{Aa}$ & $1,76 \mathrm{Aa}$ & $2,06 \mathrm{Aa}$ & $2,37 \mathrm{Aa}$ & $2,66 \mathrm{Ab}$ & $3,39 \mathrm{Aa}$ \\
\hline \multirow[t]{2}{*}{1000} & $1,58 \mathrm{Aa}$ & $1,60 \mathrm{Aa}$ & 2,17 Аа & $2,40 \mathrm{Aa}$ & $2,43 \mathrm{Ab}$ & 3,59 Aa \\
\hline & \multicolumn{6}{|c|}{ Nitrogênio na parte aérea $\left(\mathrm{g} \mathrm{kg}^{-1}\right)$} \\
\hline 0 & $14,9 \mathrm{Ab}$ & $24,3 \mathrm{ABa}$ & $19,9 \mathrm{Ab}$ & $30,7 \mathrm{Aa}$ & $18,7 \mathrm{Ab}$ & $36,0 \mathrm{Aa}$ \\
\hline 250 & $14,9 \mathrm{Ab}$ & $28,4 \mathrm{Aa}$ & $18,6 \mathrm{ABb}$ & $35,3 \mathrm{Aa}$ & $19,3 \mathrm{Ab}$ & $38,0 \mathrm{Aa}$ \\
\hline 500 & $15,8 \mathrm{Ab}$ & $25,7 \mathrm{ABa}$ & $16,9 \mathrm{BCb}$ & 35,4 Aa & $20,0 \mathrm{Ab}$ & $33,6 \mathrm{Aa}$ \\
\hline 1000 & $15,1 \mathrm{Ab}$ & $27,4 \mathrm{Ba}$ & $15,1 \mathrm{Cb}$ & $34,9 \mathrm{Aa}$ & $19,8 \mathrm{Ab}$ & $29,4 \mathrm{Aa}$ \\
\hline
\end{tabular}

Letras diferentes indicam as diferenças estatísticas pelo teste Tukey a $5 \%$. Letras minúsculas comparam C/R e S/R dentro de cada dia e letras maíusculas comparam as diferentes concentrações de $\mathrm{Pb}$, em cada tratamento. Cada dado representa o valor médio de sete repetições. 
Na matéria seca do caule em plantas com 14 dias após a aplicação de $\mathrm{Pb}$ houve diferenças significativas entre $C / R$ e $S / R$ na ausência de $\mathrm{Pb}$ e na dose de $500 \mu \mathrm{mol} \mathrm{L}^{-1}$ (Tabela 2). Em plantas com 22 dias após a aplicação de $\mathrm{Pb}$, as diferenças foram significativas na ausência de $\mathrm{Pb}$ e na dose de 250 $\mu \mathrm{mol} \mathrm{L}{ }^{-1}$. Já nas plantas com 27 dias após a aplicação $\mathrm{de} \mathrm{Pb}$, houve variação entre os tratamentos somente na dose $1.000 \mu \mathrm{mol} \mathrm{L}^{-1}$.

Na matéria seca de folhas em plantas com 14 e 22 dias após a aplicação de $\mathrm{Pb}$ houve diferenças significativas somente na ausência de $\mathrm{Pb}$ (Tabela 2). As plantas com 27 dias após a aplicação de $\mathrm{Pb}$ não apresentaram variação entre os tratamentos somente na dose de $250 \mu \mathrm{mol} \mathrm{L}^{-1}$.

Segundo Paiva (2000), em experimento com aplicações de doses crescentes de acetato de chumbo $\left(0,49,96,192\right.$ e $\left.288 \mu \mathrm{mol} \mathrm{L}^{-1}, \mathrm{pH} 5,5\right)$, verificou-se que a menor dose aplicada $\left(49 \mu \mathrm{mol} \mathrm{L}^{-1}\right)$ reduziu em $32 \%$ a massa seca da parte aérea das mudas de cedro e em $24 \%$ das mudas de ipê-roxo. Já a maior dose aplicada $\left(288 \mu \mathrm{mol} \mathrm{L}^{-1}\right)$ reduziu em mais de $70 \%$ a massa seca das duas espécies.
Com relação ao teor de $\mathrm{N}$, não foram observadas diferenças significativas em $C / R$ e $S / R$ em função do aumento da dose de $\mathrm{Pb}$ aplicada (Tabela 2). Em todos os dias analisados, na parte aérea das plantas dos tratamentos $C / R$, constataram-se menores teores de $\mathrm{N}$ que nas do tratamento $\mathrm{S} / \mathrm{R}$.

Esses resultados indicam que, embora não tenha ocorrido formação visível de nódulos, possivelmente os rizóbios auxiliaram as plantas de feijão-de-porco a manter seu teor de $\mathrm{N}$, independentemente do aumento da concentração de $\mathrm{Pb}$. Após 22 dias da aplicação de $\mathrm{Pb}$, o efeito foi contrário ao observado aos 14 dias, revelando que o $\mathrm{Pb}$ limitou a assimilação de N. Aos 27 dias após a aplicação de $\mathrm{Pb}$, o teor de $\mathrm{N}$ não variou conforme a dose de $\mathrm{Pb}$ aplicada. Em geral, no tratamento $\mathrm{S} / \mathrm{R}$ foi observado maior teor de $\mathrm{N}$ que no tratamento $\mathrm{C} / \mathrm{R}$.

O índice de produção relativa foi alto, demonstrando que o $\mathrm{Pb}$ afetou pouco a produção de matéria seca (Tabela 3). A massa verde produzida foi alta, não havendo grande impacto do $\mathrm{Pb}$ no desenvolvimento das plantas, evidenciando a possibilidade do uso do feijão-de-porco como planta fitoextratora.

Tabela 3. Índice de produção relativa (IP) em plantas de feijão-de-porco (Canavalia ensiformis (L.) D.C.) expostas a diferentes doses de $\mathrm{Pb}$ por 14, 22 e 27 dias

\begin{tabular}{|c|c|c|c|c|c|c|}
\hline \multirow{2}{*}{ Concentração de $\mathrm{Pb}$} & $\mathrm{C} / \mathrm{R}$ & $\mathrm{S} / \mathrm{R}$ & $\mathrm{C} / \mathrm{R}$ & $\mathrm{S} / \mathrm{R}$ & $\mathrm{C} / \mathrm{R}$ & $\mathrm{S} / \mathrm{R}$ \\
\hline & \multicolumn{2}{|c|}{14 dias } & \multicolumn{2}{|c|}{22 dias } & \multicolumn{2}{|c|}{27 dias } \\
\hline \multicolumn{7}{|l|}{$\mu \mathrm{mol} \mathrm{L}{ }^{-1}$} \\
\hline 250 & 97,9 a & $87,5 \mathrm{~b}$ & 99,8 a & $92,1 \mathrm{a}$ & $100 \mathrm{a}$ & $93,0 \mathrm{a}$ \\
\hline 500 & $100 \mathrm{a}$ & $97,2 \mathrm{a}$ & $100 \mathrm{a}$ & $91,5 \mathrm{~b}$ & $97,0 \mathrm{a}$ & $100 \mathrm{a}$ \\
\hline 1000 & $100 \mathrm{a}$ & $86,4 \mathrm{~b}$ & $100 \mathrm{a}$ & $91,0 \mathrm{~b}$ & $97,0 \mathrm{a}$ & $100 \mathrm{a}$ \\
\hline
\end{tabular}

Letras diferentes indicam as diferenças estatísticas pelo teste Tukey a $5 \%$, entre $\mathrm{C} / \mathrm{R}$ e $\mathrm{S} / \mathrm{R}$ dentro de cada dia, em cada concentração de Pb. Cada dado representa o valor médio de cinco repetições.

Nas raízes do tratamento $S / R$ podemos observar que aos 14 e aos 22 dias (Figuras 1A e 1B) após a aplicação de chumbo, o teor de $\mathrm{Pb}$ foi aumentando linearmente e, aos 27 dias (Figura 1C) o aumento foi quadrático. Nas raízes do tratamento C/ $\mathrm{R}$, pode-se observar que aos 14 e aos 22 dias (Figuras $1 \mathrm{~A}$ e 1B) após a aplicação de chumbo, o teor de $\mathrm{Pb}$ nas plantas de feijão-de-porco teve um aumento quadrático. Contudo, aos 27 dias (Figura 1C) após a aplicação de chumbo, o teor de $\mathrm{Pb}$ apresentou tendência de queda a partir da dose $1.000 \mu \mathrm{mol} \mathrm{L}^{-1}$.

Os teores de $\mathrm{Pb}$ detectados na parte aérea nas plantas C/R, em geral, foram bem maiores que os das $\mathrm{S} / \mathrm{R}$, em todos os dias analisados (Figura 2), apesar do menor desenvolvimento da parte aérea das plantas $\mathrm{C} / \mathrm{R}$ pelo não-suprimento de $\mathrm{N}$. Por exemplo, na concentração de $1.000 \mu \mathrm{mol} \mathrm{L}^{-1}$, aos 27 dias, no tratamento $C / R$, a massa seca da parte áerea foi de 4,01 $\mathrm{mg}$ e a quantidade de $\mathrm{Pb}$ nesta massa foi de $131,53 \mathrm{ng}$, enquanto nas plantas $S / R$, a massa seca da parte aérea foi de $5,90 \mathrm{mg}$ e a quantidade de $\mathrm{Pb}$, de $86,73 \mathrm{ng}$.

Segundo KASTORI et al. (1998), em plantas que crescem em solução nutritiva observam-se absorção positivamente correlacionada com a dose de $\mathrm{Pb}$ aplicada. Essa correlação foi observada neste trabalho, pois, à medida que a dose de $\mathrm{Pb}$ aumentou, a concentração nos tecidos também aumentou. Segundo BARTHI e Singh (1993), em raiz, caule e folha de gergelim, o acúmulo de $\mathrm{Pb}$ aumentou com as doses do metal, que variaram de 10 a $2000 \mu \mathrm{mol} \mathrm{L}^{-1}$, mas esse acúmulo foi consideravelmente maior nas raízes que nas folhas das mudas, revelando que ocorre baixa translocação do metal para as folhas. 



Figura 1. Teor de chumbo nas raízes de plantas de feijãode-porco, Canavalia ensiformis (L.) D.C., submetidas a diferentes concentrações de chumbo por 14 (A), 22 (B) e 27 (C) dias. Cada símbolo representa o valor médio de cinco repetições. (<) C/R - com rizóbios (linha contínua = polinômio $\mathrm{C} / \mathrm{R}$ ); ( ) $\mathrm{S} / \mathrm{R}$ - sem rizóbios (linha tracejada $=$ polinômio $S / R) .{ }^{* *}$ Significativo a $1 \%$.

Romeiro (2005) constatou que as doses de 200 e $400 \mu \mathrm{mol} \mathrm{L}^{-1} \mathrm{o} \mathrm{Pb}$ (acetato de $\mathrm{Pb}$ ) em hidroponia promoveram clorose e posterior necrose nas folhas mais velhas de feijão-de-porco e, em alguns casos, a queda do órgão. No presente trabalho, mesmo na dose mais alta $\left(1.000 \mu \mathrm{mol} \mathrm{L}^{-1}\right)$, não se observaram sintomas de toxicidade, ou seja, as folhas permaneceram com a mesma coloração. No entanto, à medida que as doses de chumbo aumentavam, as raízes foram escurecendo, engrossando e encurtando, confirmando resultados constatados por diferentes autores (KASTORI et al., 1998; PAIVA, 2000). KASTORI et al. (1998) supõem que esses sintomas possam ocorrer pelo fato de o metal inibir a divisão e a diferenciação celular nas raízes e, segundo Paiva (2000), esse confinamento nas raízes provoca distúrbios fisiológicos, impedindo ou dificultando o pleno desenvolvimento das plantas. No presente trabalho, este efeito não foi verificado.

Em experimento com Brassica albograta cultivada em presença de $\mathrm{Pb}$, a maior parte desse metal estava presente nas raízes (Horng et al., 2002). Wenzel e JockWer (1999) relatam haver forte habilidade das raízes em reter grandes quantidades $\mathrm{de} \mathrm{Pb}$, restringindo a translocação para a parte aérea. Em Picea abies, o teor de $\mathrm{Pb}$ nas raízes foi cerca de dez vezes maior que no caule (MARSCHNER et al., 1996).
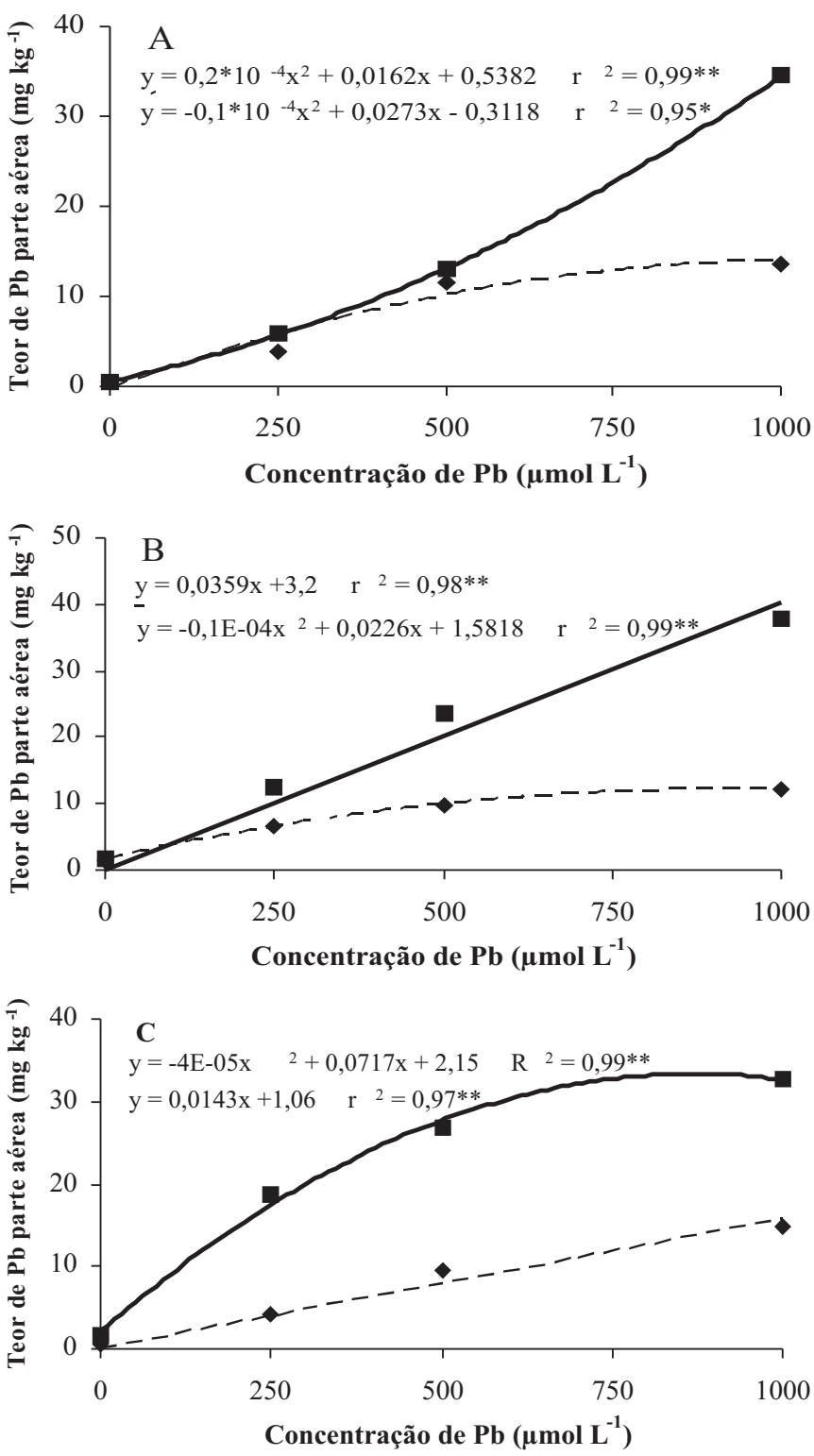

Figura 2. Teor de chumbo na parte aérea de plantas de feijão-de-porco, Canavalia ensiformis (L.) D.C., submetidas a diferentes concentrações de chumbo por 14 (A), 22 (B) e 27 (C) dias. Cada símbolo representa o valor médio de cinco repetições. (<) C/R - com rizóbios (linha contínua = polonômio $\mathrm{C} / \mathrm{R}$ ); ( ) S/R- sem rizóbios (linha tracejada $=$ polinômio $S / R)$. ${ }^{*}$ Significativo a $1 \%$. 
Verkleij e Prast (1989), afirmam que as espécies tolerantes ao $\mathrm{Pb}$ acumulam maiores concentrações nas raízes, mostrando que essas plantas não evitam a absorção do metal, mas limitam sua translocação para a parte aérea. Esta pode ser uma estratégia para o feijão-de-porco ser tolerante $\mathrm{ao} \mathrm{Pb}$, pois as quantidades deste metal na parte aérea não ultrapassaram $20 \mathrm{mg} \mathrm{kg}^{-1}$ quando houve suprimento de N que, segundo Kabata-Pendias e Pendias (1984), é o valor máximo considerado normal. Reforçando o descrito, o índice de translocação (IT) do $\mathrm{Pb}$ para as plantas de feijão-de-porco foi extremamente baixo, demonstrando que o $\mathrm{Pb}$ teve baixa mobilidade para a parte aérea (Tabela 4). Os maiores índices foram obtidos na dose de $1.000 \mu \mathrm{mol} \mathrm{L}^{-1}$ do tratamento C/R 14 dias após a aplicação de $\mathrm{Pb}$, seguido das doses de 250 e $1.000 \mu \mathrm{mol} \mathrm{L}^{-1}$ do tratamento C/R 22 dias após a aplicação de $\mathrm{Pb}$. A média do IT indica que os tratamentos $\mathrm{C} / \mathrm{R}$ obtiveram as maiores porcentagens de translocação quando comparados com os tratamentos S/R. Esses dados reforçam a hipótese de que, embora não tenha ocorrido nodulação, a aplicação de rizóbio possivelmente favoreceu a entrada e translocação do $\mathrm{Pb}$ na planta.

Tabela 4. Índice de translocação (IT) de Pb em plantas de feijão-de-porco (Canavalia ensiformis (L.) D.C.) expostas a diferentes doses de $\mathrm{Pb}$ por 14, 22 e 27 dias

\begin{tabular}{|c|c|c|c|c|c|c|}
\hline \multirow{2}{*}{ Concentração de $\mathrm{Pb}$} & $\mathrm{C} / \mathrm{R}$ & $\mathrm{S} / \mathrm{R}$ & $\mathrm{C} / \mathrm{R}$ & $\mathrm{S} / \mathrm{R}$ & $\mathrm{C} / \mathrm{R}$ & $\mathrm{S} / \mathrm{R}$ \\
\hline & \multicolumn{2}{|c|}{14 dias } & \multicolumn{2}{|c|}{22 dias } & \multicolumn{2}{|c|}{27 dias } \\
\hline \multicolumn{7}{|l|}{$\mu \mathrm{mol} \mathrm{L}{ }^{-1}$} \\
\hline 250 & $3,3 \mathrm{a}$ & $3,5 \mathrm{a}$ & $10,1 \mathrm{a}$ & $5,7 \mathrm{~b}$ & $5,7 \mathrm{a}$ & $7,5 \mathrm{~b}$ \\
\hline 500 & $4,7 \mathrm{a}$ & $4,7 \mathrm{a}$ & 8,9 a & $4,0 \mathrm{~b}$ & $7,4 \mathrm{a}$ & $8,9 \mathrm{~b}$ \\
\hline 1000 & $11,1 \mathrm{a}$ & $4,8 \mathrm{~b}$ & $12,2 \mathrm{a}$ & $4,3 \mathrm{~b}$ & $7,5 \mathrm{a}$ & $5,2 \mathrm{~b}$ \\
\hline
\end{tabular}

Letras diferentes indicam as diferenças estatísticas pelo teste Tukey a 5\%, entre C/R e S/R dentro de cada dia, em cada concentração de Pb. Cada dado representa o valor médio de cinco repetições.

O nitrogênio é considerado um dos principais nutrientes a limitar o crescimento e a produtividade dos vegetais. Plantas cultivadas com quantidades inadequadas de nitrogênio, normalmente, não expressam seu potencial produtivo, visto que, sob tais condições, podem ocorrer reduções significativas na assimilação líquida de $\mathrm{CO}_{2}$ (Evans, 1989). Neste experimento, esperava-se que o tratamento $C / R$ obtivesse maiores concentrações de $\mathrm{N}$ que o tratamento $\mathrm{S} / \mathrm{R}$. Porém, o fato de não ter ocorrido formação de nódulos radiculares, e conseqüente fixação de $\mathrm{N}_{2}$ no tratamento $C / R$, fez com que tivesse a metade da concentração de nitrogênio que no tratamento $S / R$, o qual recebeu dose extra de nitrogênio. A ausência de nodulação pode ter ocorrido devido à inibição do processo infeccioso, levando à não-formação dos nódulos (ZorNOZA et al., 2002). Esta ausência não está relacionada com a presença de $\mathrm{Pb}$, visto que mesmo na ausência de $\mathrm{Pb}$ não ocorreu nodulação.

\section{CONCLUSÕES}

1. A espécie Canavalia ensiformis (feijão-deporco) possui potencial fitoextrator para $\mathrm{Pb}$.

2. $\mathrm{O} \mathrm{Pb}$ não inibe o crescimento das plantas de Canavalia ensiformis, quando fornecido na forma de acetato até a dose de $1.000 \mu \mathrm{mol} \mathrm{L}{ }^{-1}$, não ocorrendo sintomas de fitotoxidade na parte aérea.

\section{REFERÊNCIAS}

ABICHEQUER, A.D.; BOHNEN, H. Eficiência de absorção, translocação e utilização de $\mathrm{P}$ por variedades de trigo. Revista Brasileira de Ciências do Solo, Viçosa, v. 22, p. 21-26, 1998.

ABREU, C.A; ABREU, M.F.; RAIJ, B. VAN ; SANTOS, W.R. Comparação de métodos para avaliar a disponibilidade de metais pesados em solos. Revista Brasileira de Ciência do Solo,Viçosa, v. 19, p. 463-468, 1995.

ABREU, M.F.; ANDRADE, J.C.; FALCÃO, A.A. Protocolos de análises químicas. In: ANDRADE, J.C.; ABREU, M.F. (Ed.). Análise química de resíduos sólidos para monitoramento e estudos agroambientais. Campinas: Instituto Agronômico, 2006. p.121-158.

ACCIOLY, A.M.A.; SIQUEIRA, J.O. Contaminação química e biorremediação do solo. In: NOVAIS, R.F. de; ALVAREZ, V; SCHAEFER, C.E.G.R (Ed.). Tópicos em Ciências do Solo. 1.ed. Viçosa: Sociedade Brasileira de Ciência do Solo, 2000. v. 1., p.299-352.

ATSDR - Agency for Toxic Substances and Disease Control. CERCL priority list of hazardous substances for 2003. www.atsdr.cdc.gov/cxcx3.html. Acesso em 21 de agosto de 2006.

BHARTI, N.; SINGH, R. P. Growth and nitrate reduction by Sesamum indicum cv. PB-1 responds diferentially to lead. Phytochemistry, Oxford, v. 33, n. 3, p. 531-534, 1993. 
CRUZ, J.L.; PELACANI, C.R.; CARVALHO, J.E.B.; SOUZAFILHO, L.F.S; QUEROZ, D.C. Níveis de nitrogênio e a taxa fotossintética do mamoerio "Golden". Ciência Rural, Santa Maria, v.37, n.1, p. 64-71, 2007.

EVANS, J.R. Photosynthesis and nitrogen relationship in leaves of C3 plants. Oecologia, Berlim, v.78, n.1, p.9-19, 1989.

HOAGLAND, D.R.; ARNON, D.I. The water culture method for growing plants without soils. Berkeley: California Agricultural Experimental Station, 1939. 347p.

HORNG, T.; SU-CHIACHUN; KING-VAE.; TSEN-JH; SU-CC. Heavy metals absorption of hydroponic Chinese kale. Journal of Agriculture and Forestry, Taipei, v.51, n.1, p. 73-83, 2002.

IBGE. Instituto Brasileiro de Geografia e Estatística. Disponível em: www.ibge.com.br. Acesso em 21 de agosto de 2006.

KABATA-PENDIAS, A.; PENDIAS, H. Trace elements in soils and plants. 2.ed. Boca Raton: CRC Press, 1984.315p.

KASTORI, R.; PLESNICAR, M.; SAKAC, Z.; PANKOVIC, D.; ARSENIJEVIC-MAKSIMOVIC, I. Effect of excess lead on sunflower growth and photosynthesis. Journal of Plant Nutrition, Novi Sad, Yougoslavie, v. 21, n. 1, p. 75-85, 1998.

KERBAUY, G.B. Fisiologia Vegetal. 1. ed. Rio de Janeiro: Guanabara Koogan, 2004. 452 p.

LASAT, M.M. Phytoextraction of toxic metals: a rewiew of biological mechanisms. Journal of Environmental Quality, Madison, v. 31, p. 109-120, 2002.

MALAVOLTA, E. Fertilizantes e seu impacto ambiental: micronutrientes e metais pesados - mitos, mistificação e fatos. São Paulo: Petroquímica, 1994. 153p.

MARSCHNER, P.; GODBOLD, D. L.; JENTSCHKE, G. Dynamics of lead accumulation in mycorrhizal and non-mycorrhizal Norway spruce (Picea abies (L.) Karst.). Plant and Soil, Belo Horizonte, v.178, n.2, p.239-245, 1996.

NEWMAN, L. A.; DOTY, S. L.; GERY, K. L.; HEILMAN, P. E.; MUIZNIEKS, I; Q. T. SHANG, Q. T.; SIEMIENIEC, S. T.; STRAND, S. E.; WANG, X.; WILSON, A. M. GORDON, M. P. Phytoremediation of organic contaminants: A review of phytoremediation research at the University of Washington. Journal of Soil Contamination, Seattle, v.7, p.531-542, 1998.

PAIVA, H. N. Toxidez de Cd, Ni, Pb e Zn em mudas de cedro (Cedrela fissilis Vell.) e ipê-roxo (Tabebuia impetiginosa (Mart.) Standl.). 2000. 283 f. Tese (Doutorado em Fitotecnia) Universidade Federal de Lavras, Lavras.

PIRES, F.R.; SOUZA, C.M.; SILVA, A.A.; CECON, P.R.; PROCÓPIO, S.O.; SANTOS, J.B.; FERRREIRA, L.R. Fitorremediação de solos contaminados com tebuthiuron utilizando-se espécies cultivadas para adubação verde. Planta Daninha, Viçosa, v.23, n. 4, p.711-717, 2005.

PUNZ, W.F.; SIEGHARDT, H. The response of roots of herbaceous plant-species to heavy metals. Environmental and Experimental Botany, Paris, v.33, p.85-98, 1993.
ROMEIRO, S. Potencial fitoextrator de Ricinus communis L., Helianthus annus L. e Canavalia ensiformes L. para o chumbo, em solução nutritiva. 2005. 69f. Dissertação (Mestrado em Agricultura Tropical e Subtropical)- Instituto Agronômico, Campinas, 2005.

STOLTZ, E.; GREGER, M. Accumulation properties of As, Cd, $\mathrm{Cu}, \mathrm{Pb}$ and $\mathrm{Zn}$ by four wetland plant species growing on submerged mine tailing. Environmental and Experimental Botany, Paris, v.47, n.3, p.271-280, 2002.

VERKLEIJ, J.A.C.; PRAST, J.E. Cadmium tolerance and cotolerance in Silene vulgaris (Moench.) Garcke $[=S$. cucubalus (L.) wilb.]. The New Phytologist, London, v.111, n.4, p.637$645,1989$.

VINCENT, J.M. A manual for practical study of the root-nodule bacteria. Oxford: Blackwell Scientific Publications, 1970. 164p.

VOS, J.; VAN DER PUTTEN, P.E.L. Effect of nitrogen supply on leaf growth, leaf nitrogen economy and photosynthetic capacity in potato. Field Crops Research, Amsterdam, v.59, n.1, p.63-72, 1998.

VOS, J.; VAN DER PUTTEN, P.E.L.; BIRCH, C.J. Effect of nitrogen supply on leaf appearance, leaf growth, leaf nittrogen economy and photosynthetic capacity in maize (Zea mays L.). Field Crop Research, Amsterdam, v.93, n.1, p.64-73, 2005.

ZANELLA, F. Feijão-de-porco [Canavalia ensiformis (L.) D.C.] cultivado com diferentes fontes de nitrogênio: fixação de $\mathrm{N}_{2^{\prime}}$ fotossíntese e crescimento. 2004. 76 f. Dissertação (Mestrado em Fisiologia Vegetal) - Universidade Estadual de Campinas, São Paulo.

ZORNOZA, P.; VÁZQUEZ, S.; ESTABAN, E.; FERNANDEZPASCUAL, M.; CARPENA, R. Cadmium-stress in nodulated white-lupin: strategies to avoid toxicity. Plant Physiology and Biochemistry, Amsterdam, v.40, p 1003-1009, 2002.

WENZEL, W. W.; JOCKWER. F. . Accumulation of heavy metals in plants grown on mineralized soils of the Austrian Alps. Environmental Pollution, Massachusetts, v.104, p.145155, 1999. 